\section{Launch and Deployment Analysis for a Small, MEO, Technology Demonstration Satellite}

\author{
Tyson Karl Smith ${ }^{1}$ \\ Stephen Anthony Whitmore ${ }^{2}$ \\ Utah State University, Logan, UT, 84322-4130
}

A trade study investigation the possibilities of delivering a small technology-demonstration satellite to a medium earth orbit are presented. The satellite is to be deployed in a $19,000 \mathrm{~km}$ orbit with an inclination of $55^{\circ}$. This payload is a technology demonstrator and thus launch and deployment costs are a paramount consideration. Also the payload includes classified technology, thus a USA licensed launch system is mandated. All FAAlicensed US launch systems are considered during a preliminary trade analysis. This preliminary trade analysis selects Orbital Sciences Minotaur V launch vehicle. To meet mission objective the Minotaur $\mathrm{V}^{\text {th }}$ stage ATK-Star 37FM motor is replaced with the smaller ATK- Star 27. This new configuration allows for payload delivery without adding an additional 6th stage kick motor. End-to-end mass budgets are calculated, and a concept of operations is presented. Monte-Carlo simulations are used to characterize the expected accuracy of the final orbit. An optimal launch trajectory is presented.

\begin{tabular}{|c|c|}
\hline & Nomenclature \\
\hline$a$ & $=$ semi-major axis of orbit, $\mathrm{km}$ \\
\hline$A_{\text {ref }}$ & $=$ reference area, $m_{2}$ \\
\hline ATK & $=$ Alliant Technology Systems \\
\hline CCAFB & $=$ Cape Canaveral Air Force Base \\
\hline CEA & $\begin{array}{l}\text { = Chemical Equilibrium with } \\
\text { Applications (computer program) }\end{array}$ \\
\hline CONOPS & $=$ concept of operations \\
\hline C3 & $=$ specific launch energy, $\mathrm{km}^{2} / \mathrm{sec}^{2}$ \\
\hline$E$ & $\begin{array}{l}=\text { separation spring stored potential } \\
\text { energy, joules }\end{array}$ \\
\hline e & $=$ orbit eccentricity \\
\hline EELV & $=$ evolved expendable launch vehicle \\
\hline FAA & $=$ Federal Aviation Administration \\
\hline$F_{\text {spring }}$ & $=$ spring force, $N t$ \\
\hline$G F E$ & $=$ government furnished equipment \\
\hline GPS III & $\begin{array}{l}=\text { next generation Global Positioning } \\
\text { Satellite constellation }\end{array}$ \\
\hline GTO & $=$ geostationary transfer earth orbit \\
\hline GUI & $=$ graphical user interface \\
\hline$g_{0}$ & $\begin{array}{l}=\text { acceleration of gravity at sea level, } \\
9.8067 \mathrm{~m} / \mathrm{sec}^{2}\end{array}$ \\
\hline$I$ & $=$ orbit inclination, $\mathrm{deg}$ \\
\hline
\end{tabular}

1 Graduate Student, Mechanical \& Aerospace Engineering Dept

2 Assistant Professor, Mechanical \& Aerospace Engineering Dept.

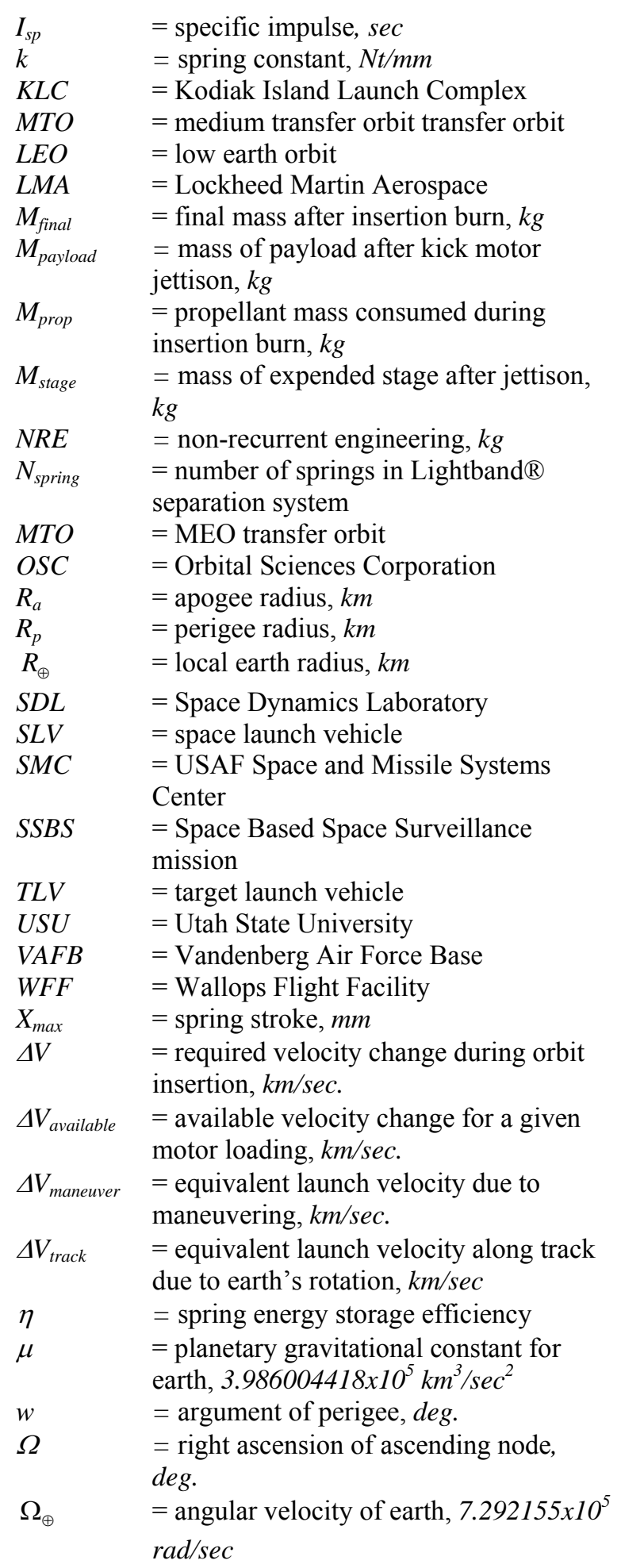

\section{Introduction}

Sandia National Laboratory is investigating advanced technologies required for nuclear explosion monitoring sensors to be deployed with the next- 
generation of Global Positioning System (GPS III) satellites. GPS III is expected to use new sensor and signal processing technologies. In order to verify that these technologies are fully developed and spacequalified, Sandia is developing a small satellite with prototype bus architecture. The satellite is to be deployed in a medium earth orbit (MEO) and have a mission life between one and three years. This satellite has been named SandiaSat. The Utah State University (USU) Department of Mechanical and Aerospace Engineering along with The Utah State University Space Dynamics Laboratory (SDL) are developing the notional mission plan, concept of operations (CONOPS), preliminary satellite and ground station designs, launch options, and associated cost estimates. This paper addresses the launch and deployment options for this mission. Fundamental mission objectives require the payload to be delivered to a circular orbit at $19,000 \mathrm{~km}$ altitude at an inclination of $55^{\circ}$. The orbit right ascension, argument of perigee, and true anomaly (phasing within the orbit) are not critical to mission. The deployment orbit was selected to be a "junk orbit" and post mission de-orbit of both the payload and expended apogee kick stage is not required. It is anticipated that the completed SandiaSat system will be available for launch during the first quarter of calendar year 2012 .

\section{Launch Vehicle Selection}

A wide array of launchers are available to deliver the payload. Many factors drive the selection of the best commercial launch systems.

In selecting a launcher for this mission the launch vehicle needs to be able to deliver the payload mass to the required orbit while allowing for sufficient mass margin. The MEO orbit selected for this study is considerably higher then the usual orbits considered for small launch systems. Usually for MEO orbits a medium-lift launch vehicle is selected, but due to the small payload mass $(<350 \mathrm{~kg})$ launchers originally used for Low Earth Orbit (LEO) were considered.

Primary constraints for the SandiaSat mission are 1) cost, 2) security, 3) launch site availability. The payload is a part of a technology demonstration and not an operational mission, thus launch and delivery costs are a paramount consideration. The payload includes classified technologies, thus the launcher is required to be a USA licensed launch system. Also the high inclination $\left(55^{\circ}\right)$ orbit favors a launch out of NASA Wallops Flight Facility (WFF). ${ }^{i}$ It is possible to reach this orbit from Cape Canaveral Air Force Base
(CCAFB), but this would require an inclination change during launch, which requires additional propellant, that in turn results in greater cost and less mass available for the payload.

A preliminary trade analysis was performed to consider every available US launch system. The preliminary trade relied on manufacturers' mission payload charts as well as impulsive $\Delta V$ calculations performed using data derived from independently published system information. Systems with launches available from WFF were given priority in the trade analysis. Launches from the west coast test range at Vandenberg Air Force Base (VAFB) are incompatible with the required orbit inclinations, and were not considered for this mission.

\section{A. Preliminary Launch Systems Trade Analysis}

The Current Federal Aviation Administration (FAA)-certified launch systems ${ }^{\text {ii }}$ licensed to carry a classified USA payload are listed below (vendor in parenthesis).

- Atlas (United Launch Alliance),

- Athena (Lockheed Martin Aerospace),

- Delta (United Launch Alliance),

- Pegasus (Orbital Sciences Corporation),

- Taurus (Orbital Sciences Corporation),

- Minotaur (Orbital Sciences Corporation),

- Falcon (Space-X),

- Space Shuttle (United Space Alliance, NASA),

- Zenit3SL (Sea Launch Odyssey LTD, MultiNational).

Figure 1 shows the characteristics of the Atlas ${ }^{\mathrm{iii}}$ family of launch systems including payload to LEO, payload to geostationary transfer orbit (GTO), launch costs in US dollars (Circa 2002), date of the first flight, and available launch sites. All of the members of this launch family have medium- or heavy-lift GTO capability, and were primarily designed for large military payloads or for sizeable geostationary communications satellite. They all possess "excess lift" capability and have launch costs that vary for \$75-110 million. For this small technologydemonstration program the costs of these systems were considered to be prohibitive.

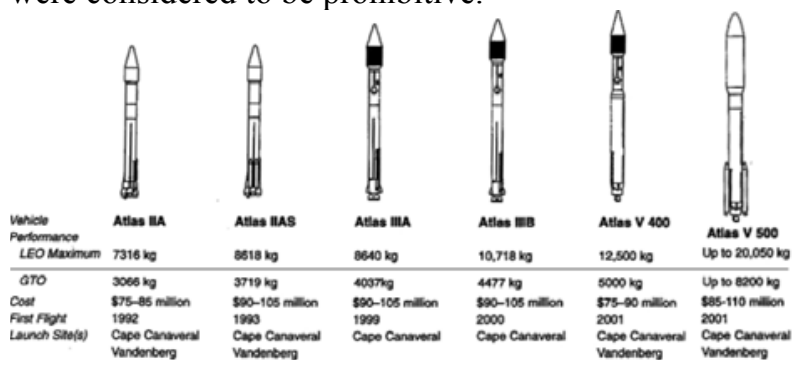


Figure 1. Atlas Series Launch Systems.

Figure 2 shows the characteristics of the Athena $^{\text {iv,v }}$ and Delta ${ }^{\text {vi }}$ family of launch systems. Payload data to LEO, to geostationary transfer orbit (GTO), launch costs in US dollars, date of the first flight, and available launch sites are all shown. The Delta III and IV systems have excess $\Delta V$ capability and launch costs that vary from $\$ 85$ to 170 million. These costs are considered prohibitive for this mission. The Athena I system has insufficient lift capability and was eliminated from consideration. The Athena II and Delta II launch systems have at least minimal lift capacity for the MEO mission, and have moderate costs varying from $\$ 22$ million (Athena II) to $\$ 60$ million (Delta II). The main concern with the Delta II launch system is the availability after 2010. An article published by in Wall Street Journal speculates about the fate of the Delta II launch system after U.S. Air Force discontinues its use of the Delta II in $2009^{\text {vii }}$ in favor of the Evolved Expendable Launch Vehicle. (EELV).

"The Delta II has been a workhorse of the U.S. space program, which has depended on the rocket and its forerunners since 1960. But the USAF command, confronting mounting war expenses and cuts in space budgets, have decided they can't afford to continue to help underwrite three Delta II launch pads, associated personnel and other fixed costs. The U.S. National Aeronautics and Space Administration seems unable to shoulder the burden of supporting the launch infrastructure on its own. The retirement of the Delta II system seems likely after the last Air Force payload is delivered in 2009.”

This uncertainly of availability makes the Delta II a high programmatic-risk option. For this single reason the Delta II, will not be considered further in this trade analysis. From this group only the Athena II will be added to the "short list" for further consideration in this trade analysis.

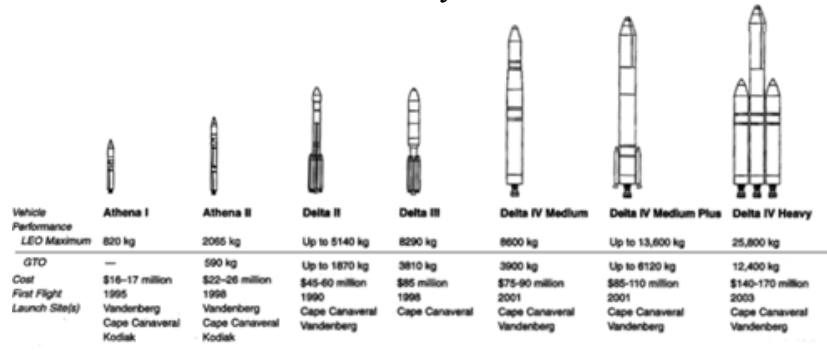

\section{Figure 2. Athena and Delta Family of Launch} Vehicles.

Figure 3 shows the characteristics of the Falcon $\mathrm{I}^{\text {viii }}$, Space Shuttle ${ }^{\mathrm{ix}}$, and Zenit $3 \mathrm{SL}^{\mathrm{x}}$ launch systems.
The Falcon 1 has insufficient lift capacity and is eliminated from consideration. The Zenit costs exceed $\$ 75$ million and combined with the launch logistics eliminated this system from consideration. During the late 1990's, riding along with the Space Shuttle as a secondary payload on one of the ISS supply missions would have been an attractive option. But with the impending retirement of the space shuttle in 2010, the shuttle was eliminated from consideration for this mission. The larger Space-X Falcon 9 medium-lift launch vehicle ${ }^{x i}$ is not considered to be of sufficient maturity to be recognized during this trade analysis.

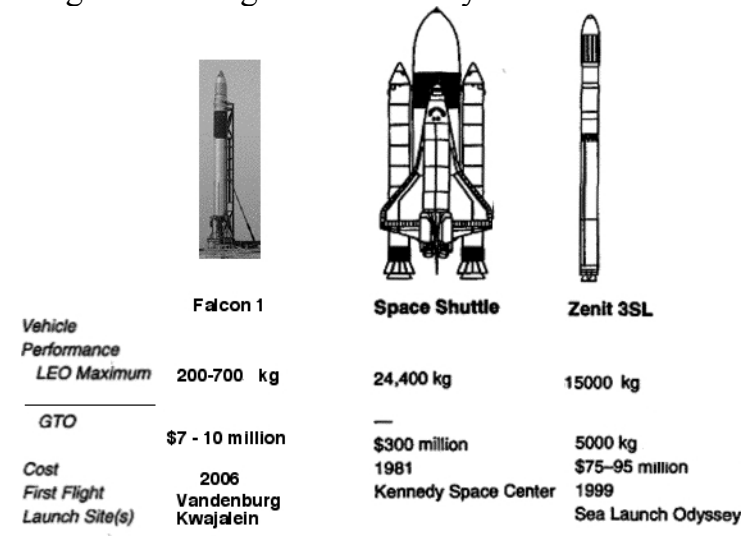

Figure 3. Falcon 1, Space Shuttle, and Zenit 3SL Launch Systems.

The family of launch vehicles from the Orbital Sciences Corporation (OSC) was also considered $^{\text {xii,xiii,xiv }}$. These launch systems are shown in Figure 4. The Minotaur I, and Pegasus XL launchers have insufficient lift capability. The Taurus XL, Minotaur IV, and Minotaur V Launch systems all have GTO capability with costs varying from $\$ 18$ million (Taurus) to $\$ 28$ million (Minotaur $\mathrm{V})^{3}$. These costs are considered to be within the scope of the allowable programmatic costs and all three systems were added to the "short list" for launch consideration. The Taurus XL is the commercially available option form the Taurus Launch system. The Minotaur IV and V launch vehicle have LEO, GTO, and escape energy configurations and variants of the same vehicle derived from the decommissioned Peacekeeper missile system.

\footnotetext{
${ }^{3}$ Verbal quote from OSC, \$35 million first flight, \$25-28 million recurrent costs thereafter.
} 


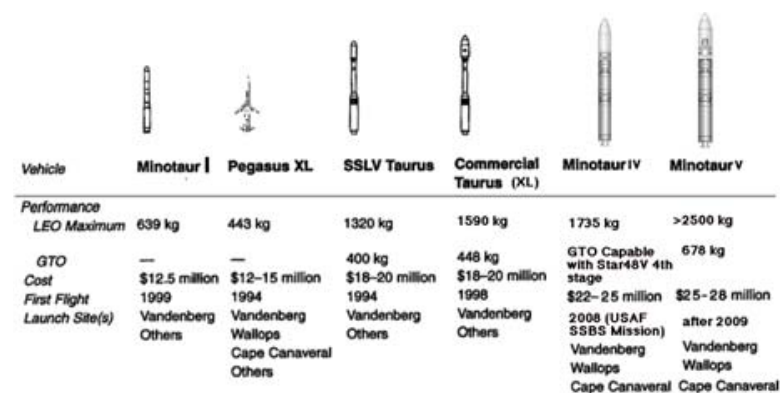

Figure 4. OSC Family of Launch Systems.

\section{B. Secondary Launch Systems Trade Analysis}

The four launcher that were placed on the acceptable "short list" included, 1) LMA Athena II, 2) OSC Taurus XL, 3) OSC Minotaur IV, and 4) OSC Minotaur $V$. The comparative positives and negatives of these systems are listed in Table 1. One of the major additional considerations is that the Athena II can only be launched from CCAFB for east coast launches. Stage impact restrictions limit the maximum inclination to $50^{\circ}$ without a plane change during the stage 3 burn. This would require a $5^{\circ}$ plane change during launch which as stated earlier adds cost and mass to the system. This loss must be considered in the "short list" trade analysis.

Table 1. Minotaur IV, Minotaur V, Athena II, and Taurus XL Launch System Comparisons.

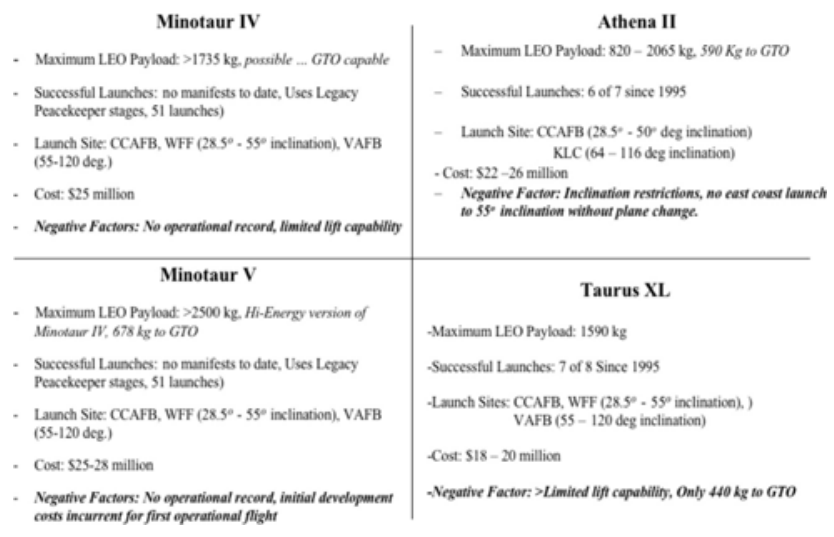

\section{Preliminary Payload Mass Budget Analysis}

Preliminary SDL designs for the SandiaSat resulted in a spacecraft mass of approximately 250 $\mathrm{kg}$. Allowing for a $35 \%$ mass contingency on the delivered payload to account for mounting structures and future mass growth, the launch system must be capable of delivering approximately $340 \mathrm{~kg}$ to the final MEO orbit. The 4 launch options listed in Table 1 must be capable of delivering, to an MTO orbit, this $340 \mathrm{~kg}$ payload plus the mass of the kick motor and propellant required to transfer from MTO to the final MEO orbit. Table 2 shows the required $\Delta V$ to transfer from a notional $1000 \times 19000 \mathrm{~km}$ altitude transfer orbit to the final $19,000 \mathrm{~km}$ altitude orbit.

Table 2. Required $\Delta V$ for MTO-to-MEO Orbit Insertion.

\begin{tabular}{|c|c|c|c|c|c|}
\hline Orbit & $\begin{array}{c}\text { Perigee } \\
\mathrm{km}\end{array}$ & $\begin{array}{c}\text { Apogee } \\
\mathrm{km}\end{array}$ & $\begin{array}{c}\text { Velocity, } \\
\mathrm{km} / \mathrm{sec}\end{array}$ & $\begin{array}{c}\text { Inclination, } \\
\text { deg. }\end{array}$ & $\begin{array}{c}\text { Required } \\
\Delta \mathrm{V}, \\
\mathrm{km} / \mathrm{sec}\end{array}$ \\
\hline MTO & 1000 & 19,000 & $\begin{array}{c}2.6597 \\
\text { (apogee) }\end{array}$ & 55 & -- \\
\hline MEO & 19,000 & 19,000 & 3.9637 & 55 & 1.3041 \\
\hline
\end{tabular}

Using the $\Delta V$ value calculated in Table 2 (1.3041 $\mathrm{km} / \mathrm{sec}$ ), Table 3 shows that a mass of $700 \mathrm{~kg}$ is required to be delivered to the MTO orbit by the launch system; a specific impulse of $285 \mathrm{sec}$, and an interstage mass of $100 \mathrm{~kg}$ are assumed. ${ }^{4}$

Table 3. Preliminary Mass to Transfer Orbit.

\begin{tabular}{|c|c|}
\hline \multicolumn{2}{|c|}{ Itemized Mass, $\mathrm{kg}$} \\
\hline Spacecraft Mass & 340 \\
\hline $\begin{array}{c}\text { Expended Kick Stage } \\
\text { +Interstage Mass }\end{array}$ & 100 \\
\hline $\begin{array}{c}\text { Required Propellant } \\
\text { for Apogee Kick }\end{array}$ & 261.6 \\
\hline $\begin{array}{c}\text { Required Mass to } \\
\text { MTO }\end{array}$ & 701.6 \\
\hline
\end{tabular}

The propellenat mass is calculated using a simple rocket equation analysis ${ }^{\mathrm{xv}}$ assuming impulsive transfer from MTO to MEO (Eqn 1)

$$
M_{\text {prop }}=M_{\text {final }} \cdot\left[e^{\frac{\Delta V}{g_{0} I_{s p}}}-1\right] .
$$

\section{C3 Payload Analysis}

A C3 launch energy analysis is done on the four launch vehicles listed in Table 1, in order to determine their lift capability. The published data for LEO, GTO and escape velocity are curve-fit and interpolated for the energy level of the MTO orbit specified in Table 2. ${ }^{\text {xii,xiii,xiv,xvi }}$ For an elliptical orbit, C3 is always a negative value. For an escape trajectory, C3 is non-negative and equals "excess hyperbolic" velocity. ${ }^{\text {xii }}$ C3 provides a convenient way to compare the required launch energies orbits with widely disparate parameters. The C3 analysis calculates the total orbital energy based on the specified orbital parameters (Eqn 2).

\footnotetext{
${ }^{4}$ While the optimized transfer orbit, kick stage $I_{s p}$, and residual mass may differ than these assumed values, these notional parameters were used to benchmark the relative lift capacity of the candidate launch systems.
} 
$C 3=-2 \cdot\left[\frac{\mu}{2 a}\right]+2 \cdot\left[\frac{\Delta V_{\text {manemering }}^{2}}{2}-\frac{\Delta V_{\text {track }}^{2}}{2}\right]=$

(Eqn 2)

$-2 \cdot\left(\frac{\mu}{R_{p}+R_{a}}\right)+\left(\Delta V_{\text {manewering }}+\Delta V_{\text {trad }}\right)\left(\Delta V_{\text {manewering }}-\Delta V_{\text {track }}\right) \approx$

$-2 \cdot\left(\frac{\mu}{R_{p}+R_{a}}\right)+\left[\Delta V_{\text {mmenenering }}+\Omega_{\oplus} R_{\oplus} \cos (i)\right] \cdot\left[\Delta V_{\text {monewering }}-\Omega_{\oplus} R_{\oplus} \cos (i)\right]$

In Eqn. $2 \mu$ is the planetary gravitational constant for the earth, $a$ is the orbit semi major axis, $R_{p}$ is the perigee radius, $R_{a}$ is the apogee radius, $\Omega_{\oplus}$ is the earth's angular velocity, $R_{\oplus}$ is the local earth radius, and $C 3$ is the specific launch energy. The term $\Delta V_{\text {maneuvering }}$ refers to any additional energy expenditures required, for example an orbital plane change. The term $\Delta V_{\text {track }}$ refers to the velocity boost along the track of the orbit provided by the earth's rotation. The final term due to earth rotation is an approximation, but is reasonably accurate as long as the orbit inclination is limited to values greater than or equal to the launch latitude.

The curve-fitted C3 data for the four launch vehicles selected are presented in Figure 5. In these figures the payload mass delivered to a given orbit is plotted against the launch energy. The solid and dashed lines represent the curve-fit data, and the single data point on each curve represents the payload mass that can be delivered to the MTO orbit at $55^{\circ}$ inclination with $1000 \times 19,000 \mathrm{~km}$ perigee and apogee altitudes. The plotted mass does not include the mass of the expended $4^{\text {th }}$ stage. The Athena II data point does include the effect of having to change orbital planes by $5^{\circ}$ during the launch.

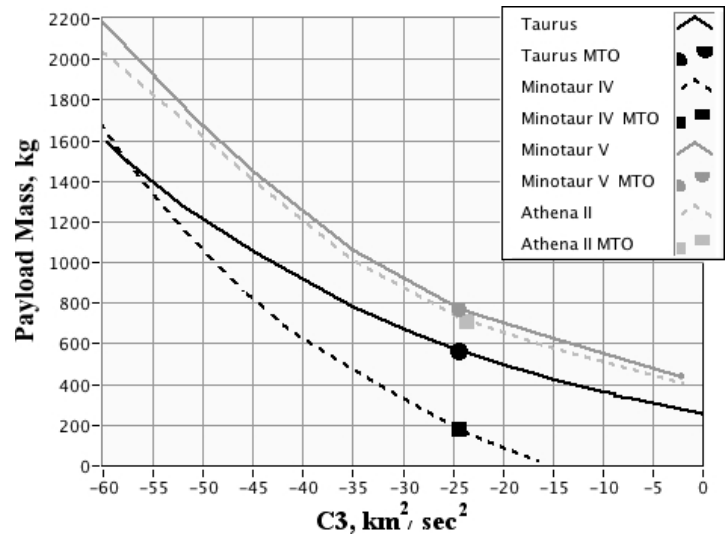

Figure 5. Launch Energy Comparisons for the 4 Candidate Launch Systems.

Table 4 summarizes the total mass that can be delivered to the MTO orbit by each of the launch systems. Only the Athena II and Minotaur V are capable of delivering the required $700^{+} \mathrm{kg}$ to the MTO. The other two systems are immediately excluded from further consideration in this analysis. The Minotaur V provides approximately $8 \%$ more lift capacity as well as having the option to launch from Wallops providing for greater launch flexibility. The availability of small payload launch windows from WFF is significantly higher than from CCAFB, and the comparative cost of launch operations is significantly lower. Thus the Minotaur V was selected as the optimal system for the SandiaSat mission.

\section{Table 4. Payload Mass Delivered to} SandiaSat MTO.

\begin{tabular}{|c|c|}
\hline Launch System & $\begin{array}{c}\text { Mass to } 1000 \times 1900 \mathrm{~km} \\
\text { altitude, } 55^{\circ} \text { inclination, } \\
\text { MTO }\end{array}$ \\
\hline Athena II & $715.4 \mathrm{~kg}$ \\
\hline Taurus $X L$ & $570.1 \mathrm{~kg}$ \\
\hline Minotaur IV & $183.5 \mathrm{~kg}$ \\
\hline Minotaur V & $775.4 \mathrm{~kg}$ \\
\hline
\end{tabular}

\section{Detailed Launch Mission Analysis}

Thus section presents a detailed launch mission analysis based on specifications and properties of the Minotaur V launch vehicle selected in the previous section. This subsection details i) the baseline Minotaur V configuration, ii) recommendations for modifications to the baseline, iii) trajectory modeling and optimization, iv) mission concept of operations, v) final mass budget analysis, vi) payload separation and re-contact analysis, vii) Monte-Carlo orbital insertion accuracy analysis, and viii) summary of the design options considered.

\section{Baseline Minotaur V Launch System}

The Minotaur family ${ }^{x i i i}$ includes the Minotaur I, IV, V space launch vehicles (SLV) and the Minotaur II and III suborbital target launch vehicles (TLV). Minotaur vehicles are available from OSC under a contract with the USAF Space and Missile Systems Center (SMC). The Minotaur IV and V SLV's are constructed using decommissioned governmentfurnished equipment (GFE) Peacekeeper missile stages $^{\text {xix }}$. These GFE stages include; stage I (TU903), stage II (SR119), and stage III (SR120). The Minotaur IV SLV adds the ATK Orion 38 motor as a $4^{\text {th }}$ stage. The Minotaur $\mathrm{V}$ is a 5-stage evolutionary version of the Minotaur IV. In the Minotaur $\mathrm{V}$ configuration the larger ATK Star 48BV replaces the Orion 38 as the $4^{\text {th }}$ stage motor. The Minotaur V also features an extended payload shroud and support structure for a fifth stage based on the ATK Star 37FM (spin-stabilized) or Star 37FMV (3-axis stabilized) kick-motor. A comparison of the Minotaur IV and $\mathrm{V}$ is shown in Figure $6 .^{\mathrm{xx}}$ 


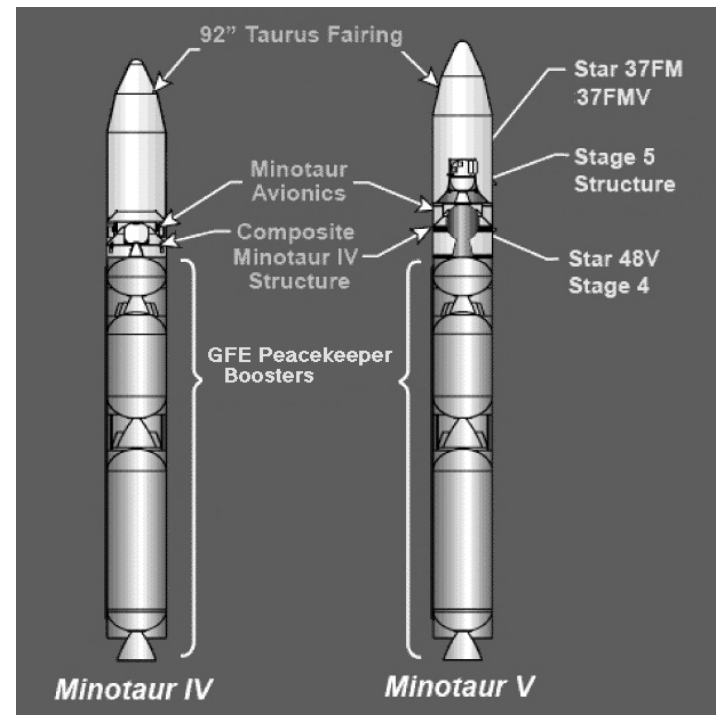

Figure 6. Comparison of the Baseline Minotaur IV and V Launch Vehicles.

There have been thirteen successful launches from the Minotaur family - seven Minotaur 1 SLVs and six Minotaur TLV's. To date neither the Minotaur IV or V systems have been launched. The Minotaur IV's maiden flight is scheduled for late 2009 and will be a USAF payload. Even though the Minotaur IV and Minotaur V launch systems have no operational history; collectively, the Peacekeeper stages I-III have successfully launched 51 times. So there is a proven flight heritage for the majority of the Minotaur IV and V sub-systems

\section{Recommended Modifications to the Baseline}

\section{Minotaur V System}

The baseline design for the Minotaur $\mathrm{V}$ is intended for high-energy missions with low negative or positive $\mathrm{C} 3$ values. Typically for these high energy missions, stages $1-5$ will be used to insert the payload into the required transfer orbit (e.g. trans-lunar injection), and a $6^{\text {th }}$ stage will be integrated with the payload for final orbit trim (e.g. lunar orbit insertion). For the lower energy MEO orbit required for the SandiaSat mission, a $6^{\text {th }}$ stage is unnecessary. A preliminary analysis based on the impulsive burn assumptions showed that the Minotaur V is capable of delivering the required satellite payload to the final MEO orbit in just 5 stages by replacing the large ATK Star 37 motor with the significantly smaller ATK Star 27 motor. This conclusion is supported by data presented in Figure 7 where the payload mass delivered to and elliptical transfer orbit by Minotaur $\mathrm{V}$ stages 1-4 is plotted against apogee altitude. Here the assumed perigee altitude for the transfer orbit is
$1000 \mathrm{~km}$, and the orbit inclination is $55^{\circ}$. The plotted mass includes the final SandiaSat payload, fully loaded apogee kick motor and any $5^{\text {th }}$ stage interstage/separation system mass. The plotted MTO payload mass does not include the assumed mass of the $4^{\text {th }}$ stage avionics module/interstage $(100 \mathrm{~kg})$ or the mass of the expended Star 48 motor. The mass delivered to an elliptical orbit with a perigee altitude of 19,000 is approximately $688 \mathrm{~kg}$. This mass is consistent with the required payload mass $(701 \mathrm{~kg})$ calculated and presented in Table 3 . This conclusion is very significant because of the potential for a less complex (and potentially less expensive) launch configuration. Direct launch simulations to be presented later in this paper will verify these preliminary impulsive rocket-equation calculations.

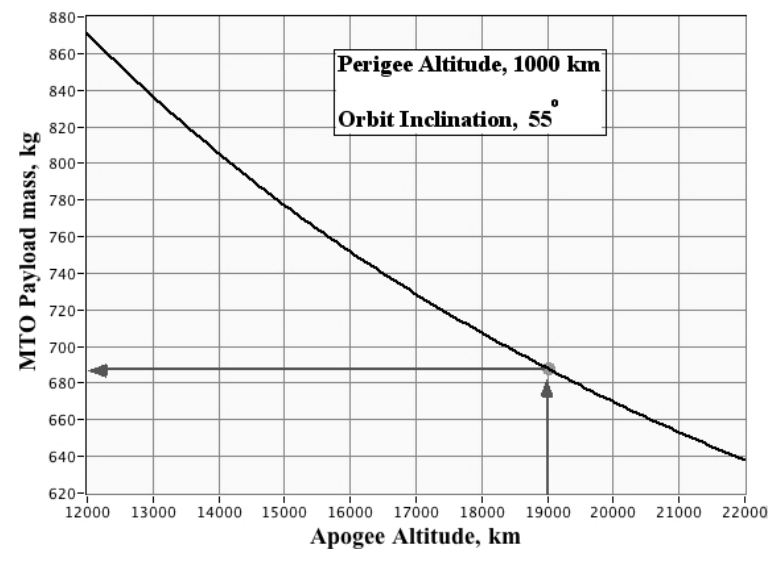

Figure 7. Payload Delivered to MTO by Minotaur V Stages 1-4.

The smaller Star 27 allows the MEO orbit to be achieved in five stages. ${ }^{\text {xxi }}$ The smaller $5^{\text {th }}$ stage also allows more working volume within the payload fairing. The Star 27 motor has a proven flight history and was developed and qualified in 1975 for use as the apogee kick motor (AKM) for the Canadian Communications Research Center Communications Technology Satellite. The Star 27 motor has served as the apogee kick motor for various applications including the NAVSTAR, GOES, and GMS series satellites. The Star 27 provides a $\Delta V$ of $1.63 \mathrm{~km} / \mathrm{sec}$ which is sufficient for final MEO orbit insertion. Recall from Table 2 a $\Delta V$ of $1.3041 \mathrm{~km} / \mathrm{sec}$ is required for final orbit insertion (Eqn 3).

$$
\Delta V=g_{o} I_{s p} \ln \left(1+\frac{M_{\text {prop }}}{M_{\text {payload }}+M_{\text {inert }}}\right)
$$

The $\Delta V$ produced by Star 27 motor is actually excessive by approximately $27 \%$ for a $340 \mathrm{~kg}$ payload mass, thus the motor may need to be off- 
loaded. The Star 27 motor can be offloaded up to $20 \%$ with out rectification, by offloading the motor the mass of the delivered payload increases. Table 5 compares the Star 37 and Star 27 motors.

Table 5. Comparison of the ATK Star 37FM and Star 27 Motors.

\begin{tabular}{|c|c|c|}
\hline Motor & Star 37 FM & Star 27 \\
\hline Total Weight & $1148.3 \mathrm{~kg}$ & $361.3 \mathrm{~kg}$ \\
\hline Length & 1.689 meters & 1.237 meters \\
\hline manufacturer & 0.935 meters & 0.693 meters \\
\hline ATK Thiokol & ATK Thiokol & ATK Thiokol \\
\hline Case & Titanium & Titanium \\
\hline Propellant & $1066.3 \mathrm{~kg}$ TP-H-3340 Solid & 333.8 kg TP-H-3135 Solid \\
\hline Nozzle & 48.2: 1 expansion ratio & 48.8: 1 expansion ratio \\
\hline $\begin{array}{l}\text { Pitch, Roll, Yaw } \\
\text { Control }\end{array}$ & ne gimbal & ne gimbal \\
\hline Burn Time & 62.7 seconds & 34.4 seconds \\
\hline Thrust & $47.276 \mathrm{kNt}$ thrust & $\begin{array}{c}25.451 \mathrm{kNt} \\
\text { thrust }\end{array}$ \\
\hline ISP & $289.8-\mathrm{sec}$ & 287.9-sec \\
\hline
\end{tabular}

\section{E. Launch Simulation and Trajectory Optimization}

A direct simulation of the proposed Minotaur V configuration with the trajectory from launch to MEO insertion was deemed necessary to verify the initial calculations presented in the previous section. The direct simulation also offers the opportunity to optimize the mission-specific endo-atmospheric portion of the launch trajectory. This three degree-offreedom, USU developed, simulation features a graphical user interface (GUI) that provides for operator interaction and direct in-the-loop control. At each data frame the vehicle pitch angle can be prescribed by direct joy-stick input, a pre-defined set of way points, or by a feedback-control loop. The interactive simulation allows for a rapid evaluation of a wide variety of candidate maneuvers and trajectories. Real-time displays allow the user to develop extensive intuition with regard to missionspecific parameters. These "piloted" simulation techniques were pioneered at NASA in early 1970's during the lifting body flight test programs and were paramount to the facilitation of this analysis. ${ }^{x i i}$ The interactive simulation also allowed perturbed conditions about the optimal trajectory for MonteCarlo analysis ${ }^{\text {xxii }}$ of expected orbit insertion accuracies.

For the $1^{\text {st }}, 2^{\text {nd }}$, and $3^{\text {rd }}$ stages, engine mass flow, nozzle exit velocity, and nozzle exit pressure were modeled to enable a thrust calculation as a function of altitude. The $4^{\text {th }}$ and $5^{\text {th }}$ stages were modeled using vacuum-thrust only. This data was collected from a variety of pubic domain sources. ${ }^{\mathrm{xxiv}, \mathrm{xxv}, \mathrm{xxvi}}$ The equilibrium gas-chemistry code Chemical Equilibrium with Applications (CEA) ${ }^{\mathrm{xxvii}}$ was used to model the combustion products based on mean properties for the specified propellants. The combustion data were used to develop the engine models for the first three stages. The aerodynamic characteristics of the first 4 vehicles stages were estimated using a panel code for subsonic flight conditions, ${ }^{x x v i i i}$ and a USU-developed incidence angle code for the supersonic flight conditions. ${ }^{x x i x, x x x}$ As the rocket motors burned base drag was assumed negligible. During stage separation and the $4^{\text {th }}$ stage coast, plume-off base drag characteristics were estimated using empirical correlations derived from Hoerner. ${ }^{\text {xxxi }}$ The launch simulation burned each of the 4 Minotaur stages to exhaustion, assuming a constant engine mass-flow, and depleting mass as a function of time. In this launch profile optimization an "apogee targeting" strategy was used where the $5^{\text {th }}$ stage and payload is inserted directly MTO orbit with a variable perigee altitude and a 19,000 km apogee altitude. After a Keplerian coast period, the $5^{\text {th }}$ stage motor was fired just before the transfer orbit apogee to insert the payload into the final orbit. Nonimpulsive, continuous-thrust calculations were used throughout the simulation. The launch trajectory optimization considered such factors as pitch profile, MTO perigee altitude, MTO orbit insertion point, and propellant offload required to match the $\Delta V$ necessary for final MEO orbit insertion. The performance metric for optimization was the final mass payload delivered to the MEO orbit.

Figure 8 shows typical simulated launch altitude (a), downrange (b), velocity (c), and acceleration (d) time histories. The extended coast time (acceleration profile) between the $3^{\text {rd }}$ stage motor burnout and the $4^{\text {th }}$ stage motor firing, this coast period was determined as a part of the trajectory optimization process. 

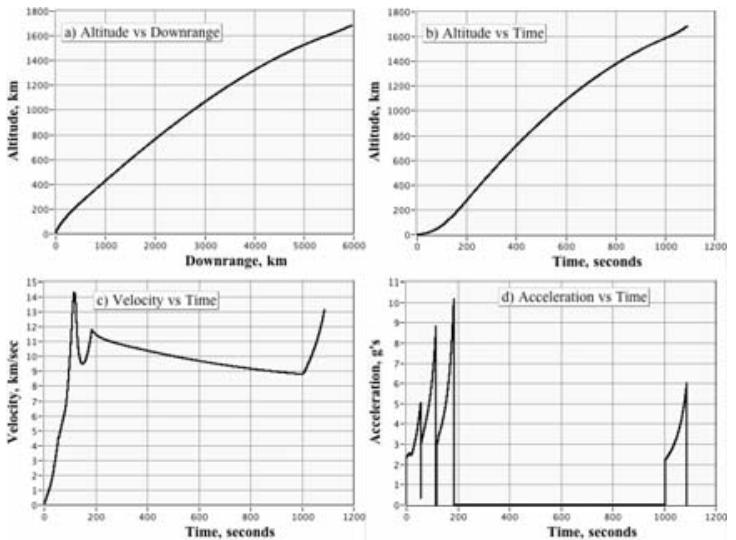

Figure 8. Typical Minotaur V MTO Insertion Launch Trajectory.

As mentioned earlier, the pitch profile and MTO insertion point were optimized to allow the maximum payload delivery. Figure 9 shows the optimized pitch profile compared against a similar ballistic trajectory for the endo-atmospheric portion of the flight; notice that the optimized trajectory initiates the "gravity turn" far sooner than on the ballistic profile and levels out once the endo-atmospheric portion of the flight is completed. This gravity turn was assisted by flying at slightly negative angles of attack to provide a downward lift on the launch stack. Figure 10 shows the lift and drag coefficient and angles-of-attack profile for the optimized launch trajectory. Lateral loads induced on the launch stack by aerodynamic forces were not considered in this optimization, but the larger negative angles-of-attack occur at higher altitudes where dynamic pressures are low and sideloads should not present a problem for the launch stack.

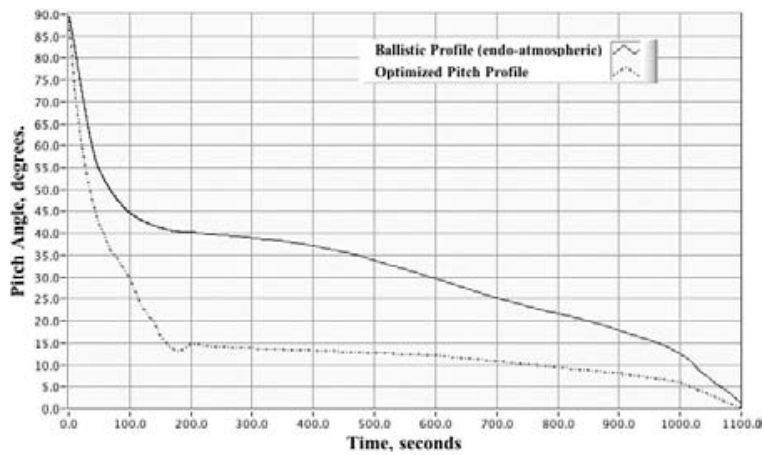

Figure 9. Optimized Launch Pitch Profile.

Figure 112 shows the optimized MTO trajectory plotted in the orbital plane ( $55^{\circ}$ inclination) and the corresponding ground track showing the launch along the descending node of the orbit. The resulting optimized transfer orbit has an apogee altitude of approximately $19,0000 \mathrm{~km}$ and a perigee altitude of approximately $1300 \mathrm{~km}$, slightly higher than the perigee altitude assumed during the preliminary trade analysis. Also the actual MTO insertion altitude is approximately $1680 \mathrm{~km}$. This insertion point into the MTO trajectory is noted on Figure 111.
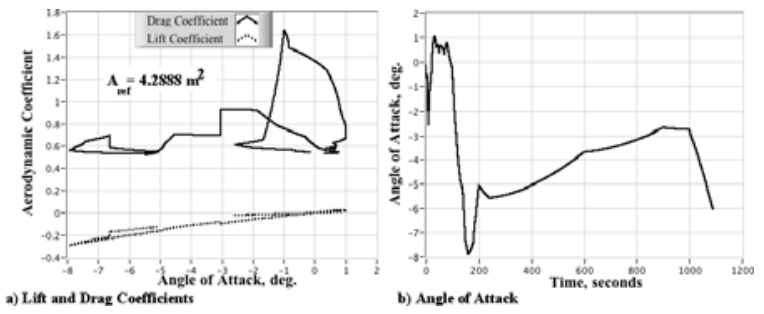

Figure 10. Aerodynamic Coefficients and Angle of Attack Along Optimized Launch Trajectory.

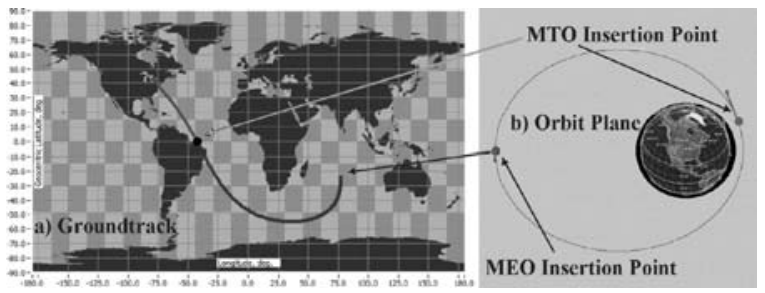

Figure 111. Optimized MTO Trajectory, Ground Track and Orbital Plane.

\section{F. Mission Concept of Operations}

An end-to-end launch and deployment concept of operations (CONOPS) is shown in Figure 13. The $4^{\text {th }}$ stage (Star 48) inserts the payload and $5^{\text {th }}$ stage (Star 27) into the MTO trajectory. The Minotaur V $4^{\text {th }}$ stage avionics module positions the payload at the required attitude and spins up the system at a rotational rate of approximately 10 revolutions/minute. The coast from MTO insertion to the orbit apogee takes approximately 2.8 hours. Once MTO apogee is reached, the Star 27 fires and inserts the payload into the circular $19,000 \mathrm{~km}$ orbit. Once inserted into the final orbit, a cold-gas jet system attached to the payload adapter cone spins down the payload. Following spin down, the expended $5^{\text {th }}$ stage is separated from the SandiaSat. Total mission elapsed time from launch to solar panel deployment is approximately 3.16 hours. 


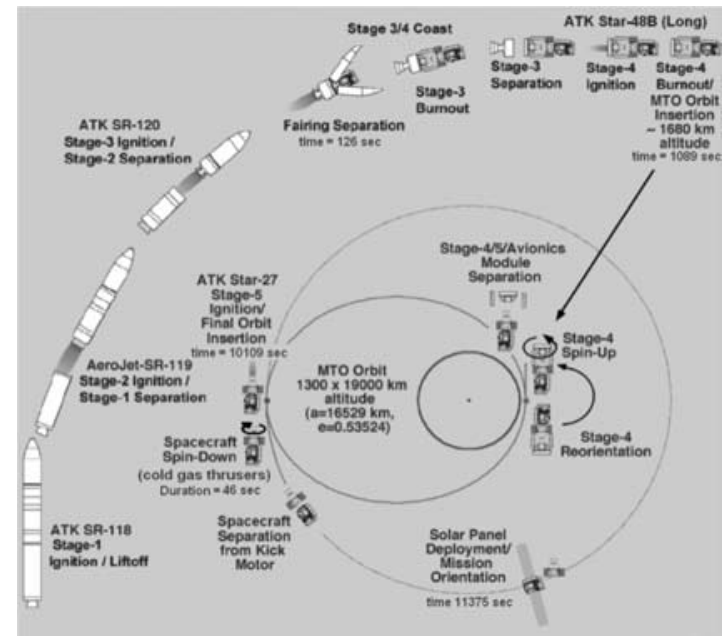

Figure 123. Launch and Deployment Concept of Operations.

\section{G. Mass Budget Analysis}

As previously mentioned the Star 27 provides excess $\Delta V$ for the orbit insertion. Consequently, off loading propellant allows more payload mass to be delivered to the final orbit. The required apogee kick $\Delta V$ (and hence the propellant off-load for the Star 27 $5^{\text {th }}$ stage) depends on the precise MTO trajectory energy level reached during the launch and MTO insertion phase of the mission. The $4^{\text {th }}$ stage payload (satellite and $5^{\text {th }}$ stage) mass delivered to MTO is approximately $682 \mathrm{~kg}$. Table 6 shows the Stage IV mass budget resulting from the optimized MTO launch analysis. The delivered mass of approximately $682 \mathrm{~kg}$ into the $55^{\circ}$ inclination MTO trajectory from WFF is comparable to the mass value presented in Table 4 that was calculated earlier using the impulsive analysis.

\section{Table 6. Mass Budget for Optimized Launch and} MTO Insertion.

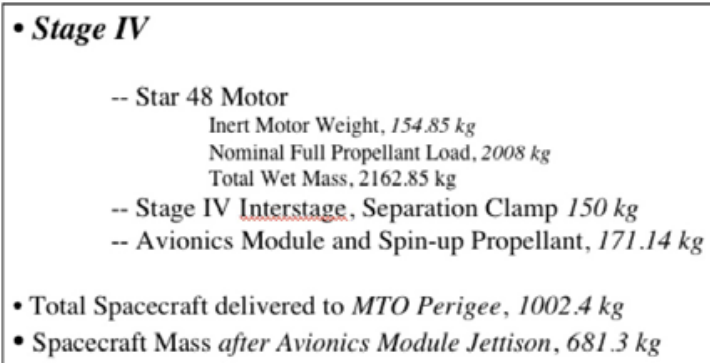

- Total Spacecraft delivered to MTO Perigee, $1002.4 \mathrm{~kg}$

- Spacecraft Mass after Avionics Module Jettison, $681.3 \mathrm{~kg}$

Table 7 shows the mass budget for the MEO insertion. The Total Spacecraft mass allocation is approximately $360 \mathrm{~kg}$ and is considered to be the maximum that the modified Minotaur V SLV can deliver to the required $19,000 \mathrm{~km}$ orbit, $55^{\circ}$ inclination MEO orbit without a $6^{\text {th }}$ stage kick motor.
The $360 \mathrm{~kg}$ maximum payload offers a $40 \%$ contingency for mass growth. A $26.4 \%$ propellant offload of the Star 27 is required for MEO orbit insertion.

\section{Table 7. Mass Budget for Optimized MEO} Insertion.

\begin{tabular}{|c|}
\hline - Stage $\boldsymbol{V}$ \\
- Total Spacecraft delivered to MTO Apogee, $681.3 \mathrm{~kg}$ \\
-- Lightband Separation System \\
Upper ring, $2.06 \mathrm{~kg}$ \\
Lower Ring, $4.11 \mathrm{~kg}$ \\
\# of Separation Springs, 26 \\
\# of Separation Connectors and Switches, 12 \\
-- Star 27 Kick Motor \\
Inert Motor Weight, $27.5 \mathrm{~kg}$ \\
Nominal Full Propellant Load, $333.76 \mathrm{~kg}$ \\
Propellant Offload, 26.4\% \\
-- Kick Motor Adaptor Cone, $16 \mathrm{~kg}$ \\
-- Cold Gas Spin--Down System, $25 \mathrm{~kg}$ \\
- Total Spacecraft Mass Delivered to MEO, $436.5 \mathrm{~kg}$ \\
- Spacecraft Mass after Kick Motor Separation, $362.4 \mathrm{~kg}$ \\
- Delivered MEO Spacecraft Mass Allocation, $360.4 \mathrm{~kg}$ \\
\hline
\end{tabular}

\section{H. Payload Separation and Re-contact Analysis}

The Planetary Systems Corporation ${ }^{\text {xxxii }} 38^{\prime}$ "

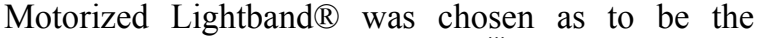
separation system on SandiaSat. ${ }^{\text {xxiii }}$ The Lightband is stowed with links locking a retaining ring that holds the separation system together. Motors drive a mechanism that allows the retaining ring to contract. The contracted ring allows spring plungers to disengage the payload side of the ring, and separation springs push the two rings apart. The separation springs impart $\Delta V$ to the payload, separating it from the expended $5^{\text {th }}$ stage motor. The resulting separation $\Delta V$ is determined by the number of separation springs installed in the system. The resulting separation $\Delta V$ is described by Eqn 4 .

$$
\Delta V=\sqrt{k \eta X^{2}{ }_{\text {max }} N_{\text {spring }}\left(\frac{M_{\text {payload }}+M_{\text {stage }}}{M_{\text {payload }} M_{\text {stage }}}\right)}
$$

In Eq. $4 \Delta V$ is the relative velocity between the payload mass ( $\left.M_{\text {payload }}\right)$ and the expended stage mass $\left(M_{\text {stage }}\right) . \quad \eta$ is the spring potential energy storage efficiency, $k$ is the spring constant, and $X_{\max }$ is the full stroke of the separation spring. Table 8 shows the separation spring data published by Planetary Systems. ${ }^{\text {xxiv }}$

Table 8. 38" Lightband Separation Spring Parameters . 


\begin{tabular}{|c|c|c|c|c|}
\hline $\begin{array}{c}\text { Spring } \\
\text { Efficiency } \\
\eta\end{array}$ & $\begin{array}{c}\text { Spring } \\
\text { Constant, } \\
k\end{array}$ & $\begin{array}{c}\text { Full } \\
\text { Stroke, } \\
X_{\max }\end{array}$ & $\begin{array}{c}\text { Spring } \\
\text { Force, } F_{\text {spring }} \\
\text { Compressed }\end{array}$ & $\begin{array}{c}\text { Spring } \\
\text { Energy, } E \\
\text { Compressed }\end{array}$ \\
\hline$\sim 0.9$ & $\begin{array}{c}4.08 \\
\mathrm{Nt} / \mathrm{mm}\end{array}$ & $\begin{array}{c}21.06 \\
\mathrm{~mm}\end{array}$ & $85.93 \mathrm{Nt}$ & $0.9048 \mathrm{~J}$ \\
\hline
\end{tabular}

Because springs store energy inefficiently when compared to pyrotechnic-based separation system, they produce only small separation velocities. The cost of spring mass must be traded against the required separation velocity and recontact after separation is a potential issue. The 38 " Motorized Light Band allows a maximum of 94 separation springs. Assuming a spring efficiency ( $\eta$ ) of 0.9 , the maximum 94 separation springs provides a separation $\Delta V$ of $1.58 \mathrm{~m} / \mathrm{sec}$. This system with 94 springs is very stiff and a separate mechanism would be needed to compress the springs during the payload installation. The additional number of springs also takes away from available satellite mass growth. A compromise value of 26 springs was selected. This number of spring results in a total Lightband resistance force of $2234 \mathrm{~N}$. This resistance force can be easily compressed by the weight of the satellite and makes for easier processing during payload installation. The 26 separation springs provide a separation $\Delta V$ of 1.05 $\mathrm{m} / \mathrm{sec}$.

If the separation is delayed for 120 seconds after burnout of the Star 27 motor to insure that residual propellant burning is completed, simulations show that the 26-spring $\Delta V$ is sufficient to prevent recontact between the payload and exhausted Star 27 upper stage. Figure 133 shows the separation distance between the SandiaSat payload and the expended $5^{\text {th }}$ stage following jettison. This analysis assumes that the Lightband separation $\Delta V$ is directed with a $5^{\circ}$ degree pitch angle and a $5^{\circ}$ out-of -plane (yaw) angle during separation. The absolute separation distance in $\mathrm{km}$ is plotted with a logarithmic scale on the ordinate and the elapsed time from the MEO insertion burn in years is plotted on the abscissa. As shown in Figure 13 a possible recontact happens every two years. Over the maximum lifetime of the mission the closest approach is greater then $20 \mathrm{~km}$ It is possible that somewhere beyond the lifetime of the SandiaSat satellite the two objects could recontact creating an orbital-debris scenario. But since the SandiaSat MEO orbit is considered a "junk orbit" this is not a great concern.

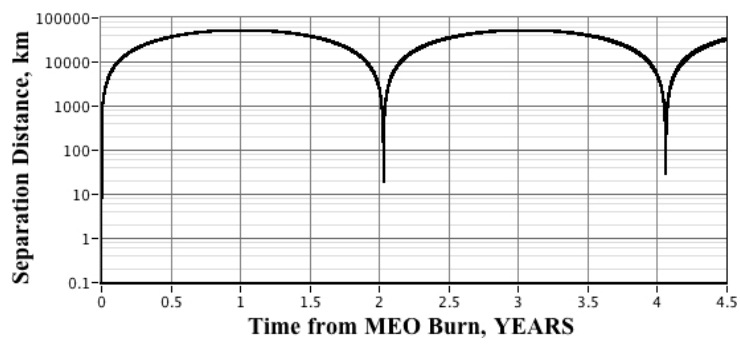

Figure 133. Time History of Separation Distance Between Payload and Expended 5th Stage.

\section{Monte Carlo Analysis of Expected Orbit}

\section{Insertion Accuracy}

The end-to-end final orbit insertion accuracy was estimated using a Monte-Carlo simulation analysis. For this analysis, the interactive simulation kernel was run in a batch-mode with rocket and orbit parameters perturbed using Gaussian white noise models. Table 9 shows the 1- $\sigma$ noise inputs to the Monte-Carlo model. A total of 997 data runs were performed to establish statistical validity (Ref. xxiii), and statistics of the final orbital parameters were calculated. Table 10 shows the end-to-end uncertainty estimates in the final MEO parameters. These uncertainties also include the effects of the Lightband separation $\Delta V$.

Table 9. 1- $\sigma$ Uncertainty Models in End-to-End Monte Carlo Simulation

- Characteristics Perturbed for Launch and Final Orbit Insertion

- Launch Error Sources, 1- $\sigma$

-- Pitch Profile, $\pm 0.05 \%$ total error (deviation from optimal) .. assumes pitch guidance feedback

-- Uncertainty in Drag Coefficient, $\pm 5 \%$

-- Uncertainty in Lift Coefficient, $\pm 5 \%$

- Thrust Uncertainty, $\pm 0.1 \%$

- Burn Time Uncertainty, $\pm 0.1 \%$

-- Total Impulse Uncertainty, $\pm 0.1414 \%$

- Kick Motor Insertion I (Star 27) Uncertainties (from ATK)

-- Thrust Error, $0.16667 \%$

- Burn Time Uncertainty, $0.084 \%$

-- Total Impulse Uncertainty, $0.1867 \%$

-- Burn Pitch Misalignment Uncertainty, $\pm l^{\circ}$

-- Burn Out-of-Plane Misalignment Uncertainty, $\pm 1^{\circ}$

- Lightband Separation Uncertainties

-- Separation $\Delta V$ Uncertainty, $\pm 0.1 \mathrm{~m} / \mathrm{sec}$

-- Separation Pitch Misalignment Uncertainty, $\pm l^{\circ}$

-- Separation Out-of-Plane Misalignment Uncertainty, $\pm l^{\circ}$

Table 10. End-to-End Uncertainty Estimates in Final MEO Orbit Parameters

\begin{tabular}{|l|l|l|}
\hline Parameter & Mean Value & $\mathbf{1}$ - $\boldsymbol{\text { Stnd. Dev. }}$ \\
\hline$a, \mathrm{~km}$ & 25370.2 & +80.8 \\
\hline$e$ & 0.00124 & +0.0004 \\
\hline i, deg & 54.991 & +0.115 \\
\hline$\Omega, \mathrm{deg}$ & 197.71 & +0.080 \\
\hline
\end{tabular}




\begin{tabular}{|l|l|l|}
\hline$\omega$, deg & 151.25 & +0.119 \\
\hline Perigee, $\mathrm{km}$ & 18975.8 & +83.9 \\
\hline Apogee, $\mathrm{km}$ & 19022.9 & +86.2 \\
\hline
\end{tabular}

\section{J. Design Change Summary}

The trade options and the effects of these changes to the MEO launch analysis is shown in Table 11. The major design consideration was the replacement of the Star 38/FMV $5^{\text {th }}$ stage on the Minotaur V with the smaller Star 27 motor. This design change enables the Minotaur V launch system to deliver the SandiaSat payload to the required MEO orbit without using a $6^{\text {th }}$ stage kick motor.

Table 11. Design Changes and Options Considered.

\begin{tabular}{|l|l|l|c|}
\hline \multicolumn{1}{|c|}{$\begin{array}{c}\text { Design Baseline } \\
\text { Change Option }\end{array}$} & Technical Performance Variation & $\begin{array}{l}\text { Programmatic } \\
\text { Cost Variation }\end{array}$ & $\begin{array}{c}\text { Programmatic } \\
\text { Schedule } \\
\text { Variation }\end{array}$ \\
\hline $\begin{array}{l}\text { Minotaur V with Star } \\
\text { 37 upper stage }\end{array}$ & $\begin{array}{l}\text { Star 37 too massive for MEO } \\
\text { insertion, No residual payload }\end{array}$ & & Minimal \\
\hline $\begin{array}{l}\text { Minotaur V with Star } \\
27 \text { upper stage }\end{array}$ & $\begin{array}{l}\text { With 25-30\% offload, 380+ kg } \\
\text { deliverable to MEO }\end{array}$ & 5250K increase & Minimal \\
\hline $\begin{array}{l}26 \text { spring Lighthand } \\
\text { replaces original 92 } \\
\text { spring option }\end{array}$ & None & minimal & Minimal \\
\hline
\end{tabular}

\section{Summary and Concluding Remarks}

A trade study investigating the economics, mass budgets, and concept of operation for delivery of a small, technology demonstration satellite to a medium-altitude earth orbit is presented. Sandia National Laboratory has proposed this prototype satellite to space-test and mature emerging technologies required for the next generation of global positioning satellites. A primary objective is the maturation low readiness technologies required for nuclear explosion monitoring. Mission requirements specify the payload to be delivered to a circular orbit at 19,000 km altitude and an inclination of $55^{\circ}$. The payload includes classified technologies and a USA licensed launch system is mandated. A preliminary trade analysis is performed where all available US launch systems are considered. The initial trade study identifies the Minotaur V launch system is the best launch option. The Minotaur V is a 5 -stage evolutionary version of the Minotaur IV constructed using decommissioned governmentfurnished (GFE) Peacekeeper missile stages for the first three stages. End-to-end mass budgets are calculated, and a concept of operations is presented. Monte-Carlo simulations are used to characterize the expected accuracy of the final orbit. An optimal launch trajectory is presented.

The primary conclusion of this study is that replacing the baseline $5^{\text {th }}$ stage ATK-37FM motor by the significantly smaller ATK Star 27 allows the final orbit to be reached without a $6^{\text {th }}$ stage. This result is very significant in that it offers a less complex and potentially less expensive launch configuration, and provides significantly more working volume within the payload fairing. Other significant conclusions are:

i) Optimized trajectory delivers a total of $1002.4 \mathrm{~kg}$ to MTO (681 kg minus inert $4^{\text {th }}$ stage),

ii) Star 27 kick motor requires $\sim 25-30 \%$ offload for proper payload insertion $\Delta \mathrm{V}$,

iii) Total spacecraft mass after kick motor separation is approximately $360 \mathrm{~kg}$, a $40 \%$ mass margin,

iv) Lightband with 26 separation springs provided sufficient DV to avoid recontact for mission lifetime,

v) Monte-Carlo analysis shows 1- $\sigma$ apogee/perigee accuracy of approximately $19000 \pm 85 \mathrm{~km}$,

vi) Monte-Carlo analysis shows final orbit inclination of approximately $55^{\circ} \pm 0.1^{\circ}$,

viii) Total mission time line from launch to final orbit is approximately 3.16 hours,

i Chiulli, Roy M., International Launch Site Guide, The Aerospace Press, El Segundo, CA, 1994, Chap. 13.

ii Isakowitz, Stephen J., Hopkins, Joseph P., Jr., and Hopkins, Joshua B., International Reference Guide to Space Launch Systems, 4th ed., American Institute of Aeronautics and Astronautics, Reston, VA, 2003, Chaps. 2, $5,6,8,15,25,28,32$.

iii Atlas, Encyclopedia Astronautica, http://www.astronautix.com/lvfam/atlas.htm, [retrieved 01 Dec. 2007].

iv Athena 1, Encyclopedia Astronautica, http://www.astronautix.com/lvs/athena1.htm, [retrieved 01 Dec. 2007].

v Athena 2, Encyclopedia Astronautica, http://www.astronautix.com/lvs/athena2.htm, [retrieved 01 Dec. 2007].

vi Delta, Encyclopedia Astronautica, http://www.astronautix.com/lvs/delta.htm, [retrieved 01 Dec. 2007].

vii DeltaII's Fate Worries Nonmilitary Users, Wall Street Journal On-line, http://online.wsj.com/article/SB118039764439516631.html ?mod=googlenews_ws], May 29, 2007, [retrieved 3 Dec. 2007].

viii Falcon 1 Overview, Space Exploration Technologies, http://www.spacex.com/falcon1.php, [retrieved 02 Dec. 2007]. 
ix Space Shuttle, Encyclopedia Astronautica, http://www.astronautix.com/craft/spauttle.htm, [retrieved 02 Dec 20007].

x Zenit 3SL, Encyclopedia Astronautica, http://www.astronautix.com/lvs/zenit3sl.htm, [retrieved 02 Dec 20007].

${ }^{x i}$ Falcon 9 Overview, Space Exploration Technologies, http://www.spacex.com/falcon9.php, [retrieved 02 Dec. 2007].

xii Taurus Launch System Payload User's Guide, Release 4.0, Orbital Sciences Incorporated, March 2006, http://www.orbital.com/NewsInfo/Publications/taurus-userguide.pdf, [Retrieved 15 May, 2007.]

xiii Minotaur IV User's Guide, Release 1.1, Orbital Sciences Incorporated, January 2006, http://www.orbital.com/NewsInfo/Publications/Minotaur_ I V Guide.pdf, [Retrieved 15 May 15, 2007.]

xiv Minotaur V Fact Sheet, http://www.orbital.com/NewsInfo/Publications/Minotaur $\mathrm{V}$ fact.pdf, Orbital Sciences Incorporated, [Retrieved 02 Dec. 2007).

${ }^{\mathrm{xv}}$ Sellers, Jerry Jon, Understanding Space: An Introduction to Astronautics, 2nd Edition, McGraw Hill, New York, 2000, Chaps. 7, 9.

xvi Mosher, Larry, E., "Solar Terrestrial Relations Observatory (STEREO), Pre-Phase-A Requirements Review, Launch Vehicle," John Hopkins University, http://stereo.nrl.navy.mil/orig_stereo/PPA/PPA_RR2_secti on 6.pdf, [Retrieved 03 Dec. 2007].

${ }^{\text {xvii }}$ Vallado, David A., Fundamentals of Astrodynamics and Applications, $2^{\text {nd }}$ ed., Microcosm Press, El Segundo, CA, 2001, Chaps. 1,6.

xviii Schoneman, Scott, Amorosi, Lou, Cheke, Dan, and Chadwick, Mark, "Minotaur V Space Launch Vehicle for Small, Cost-Effective Moon Exploration Missions," AIAA SSC07-III-2, Presented at $21^{\text {st }}$ AIAA/USU Conference on Small Satellites, Logan UT, August, 2007.

xix Peacekeeper Missile System, http://www.geocities.com/peacekeeper_icbm/specs.htm, [Retrieved August 15, 2007].

${ }^{x x}$ Minotaur Launch System, Encyclopedia Astronautica, http://www.astronautix.com/engines/peaeper1.htm,

[Retrieved August 15, 2007].

xxi Star 37 Motor, ATK Star Motor Overview, Alliant Tech Systems, http://www.mission.com/starmotors/starmotors_star37.asp, [Retrieved: 6 October, 2007]. xxii Evans, M. B., and Schilling, L., J., "The Role of Simulation in the Development and Flight Test of the HIMAT Vehicle," NASA-TM-84912, April 1984.

xxiii Sobol Ilya M., A primer for the Monte Carlo Method, CRC Press, Boca Raton, FL, 1994, Chaps 1-3.

xxiv Peacekeeper Missile, http://www.geocities.com/peacekeeper $\mathrm{icbm} / \mathrm{specs.htm}$, Retrieved: 15 August, 2007.,

$\mathrm{xxy}$

Encyclopedia

Astronautics,

http://www.astronautix.com/engines/peaeper1.htm,

Retrieved: 15 August, 2007.

xxvi ATK Launch and Mission Systems, Alliant Tech Systems,

http://www.mission.com/starmotors/starmotors star48v.asp , Retrieved: 15 August, 2007.

xxvii Gordon, S., and McBride, B. J., "Computer Program for Calculation of Complex Chemical Equilibrium Compositions and Applications”, NASA RP-1311, 1994.

xxviii AEROCFD, Apogee Rockets, http://www.apogeerockets.com, Retrieved: 7 October, 2007.

xxix Whitmore, S. A., "Conical Supersonic Flow Fields," Utah State University, URL:

http://www.engineering.usu.edu/classes/mae/5420/Compre ssible fluids/section11.html, Retrieved: 15 November, 2005

xxx Anderson, J. D. Jr., Modern Compressible Flow with Historical Perspective, $3^{\text {rd }}$. ed., McGraw-Hill Higher Education, New York, 2003, Chapter 4.

xxxi Hoerner, Sighard F., Fluid Dynamic Drag, SelfPublished Work, Library of Congress Card Number 641966, Washington, D.C., 1965, pp. 3-19, 3-20.

xxxii Lightband Separation Systems, Planetary Systems Incorporated, http://www.planetarysystemscorp.com, Retrieved: 6 October, 2007.

xxxiii Holemans, Walter, "The Lightband as Enabling Technology for Responsive Space," AIAA Paper RS22004-7005, Presented at the Second Annual Responsive Space Conference, Los Angeles, April, 2004.

xxxiv Anon., “2000875 Rev - User's Manual for Mark II Lightband," Planetary Systems Incorporated, http://www.planetarysystemscorp.com/download/2000785UserManual.pdf, [Retrieved 05 Dec. 2007]. 